\title{
Penetration of blood-brain barrier and antitumor activity and nerve repair in glioma by doxorubicin- loaded monosialoganglioside micelles system
}

This article was published in the following Dove Press journal:

International Journal of Nanomedicine

7 July 2017

Number of times this article has been viewed

\section{Dan Zou' \\ Wei Wang' \\ Daoxi Lei' \\ Ying Yin' \\ Peng Ren' \\ Jinju Chen ${ }^{2}$ \\ Tieying Yin' \\ Bochu Wang' \\ Guixue Wang' \\ Yazhou Wang'}

'Key Laboratory of Biorheological Science and Technology, Ministry of Education, College of Bioengineering, Chongqing University, Chongqing, People's Republic of China; ${ }^{2}$ School of Mechanical and System Engineering, Newcastle University, Newcastle Upon Tyne, UK
Correspondence: Yazhou Wang Key Laboratory of Biorheological Science and Technology, Ministry of Education, College of Bioengineering, Chongqing University, No 174 Shazheng Street, Shapingba District, Chongqing, 400044, People's Republic of China

Tel +86 I58 03055646

Email yazhou_wang@cqu.edu.cn
Abstract: For the treatment of glioma and other central nervous system diseases, one of the biggest challenges is that most therapeutic drugs cannot be delivered to the brain tumor tissue due to the blood-brain barrier (BBB). The goal of this study was to construct a nanodelivery vehicle system with capabilities to overcome the BBB for central nervous system administration. Doxorubicin as a model drug encapsulated in ganglioside GM1 micelles was able to achieve up to $9.33 \%$ loading efficiency and $97.05 \%$ encapsulation efficiency by orthogonal experimental design. The in vitro study demonstrated a slow and sustainable drug release in physiological conditions. In the cellular uptake studies, mixed micelles could effectively transport into both human umbilical vein endothelial cells and C6 cells. Furthermore, biodistribution imaging of mice showed that the DiR/GM1 mixed micelles were accumulated sustainably and distributed centrally in the brain. Experiments on zebrafish confirmed that drug-loaded GM1 micelles can overcome the BBB and enter the brain. Among all the treatment groups, the median survival time of C6-bearing rats after administering DOX/GM1 micelles was significantly prolonged. In conclusion, the ganglioside nanomicelles developed in this work can not only penetrate BBB effectively but also repair nerves and kill tumor cells at the same time.

Keywords: blood-brain barrier, GM1, nanovesicles, doxorubicin, glioma, zebrafish

\section{Introduction}

Previous studies have showed that over $80 \%$ of the intracranial tumors are malignant gliomas, which are essentially incurable and have a high rate of recurrence. ${ }^{1,2}$ Glioblastoma is the most frequent and malignant brain tumor. ${ }^{3}$ Currently, the standard treatment for patients diagnosed with glioblastoma is temozolomide chemoradiotherapy postsurgery. ${ }^{4}$ Other chemotherapeutics have little effects because of the inherent resistance of intracranial microenvironment against most cytotoxic drugs and the existence of blood-brain barrier (BBB) ${ }^{5}$ which consists of endothelial cells combined with tight intracellular junctions, pericytes, and astrocytes. ${ }^{6}$ The BBB, a diffusion barrier with selective permeability between blood and brain, can protect the normal function of the central nervous system (CNS). Meanwhile, approximately $100 \%$ of macromolecular drugs and over $98 \%$ of small-molecule compounds do not cross BBB and reach the brain tissue. ${ }^{5}$ Therefore, there is a growing need to develop safe and effective methods to deliver BBB-impermeable drugs into the brain.

Recently, there is a significant advance in the development of nanotechnologybased pharmaceutical products. ${ }^{7}$ Nanomaterials, such as polymeric nanoparticles (NPs), liposomes, dendrimers, and micelles, are focused on overcoming BBB and enhancing 
drug delivery. ${ }^{8}$ However, various potential risks, such as physicochemical properties and uncertain degradation process of carrier materials, have the possibility of destroying the normal physiological functions of CNS. Consequently, it is crucial to choose appropriate carrier materials or construct nano-drug delivery systems.

Different from other types of tumors, glioma presents space-occupying lesions, which increase intracranial pressure and lead to secondary brain edema. The insertion of the tumor into brain tissue which leads to abnormal stress, as well as aggressive tumor growth would further trigger focal brain damage and compression of normal brain tissue. ${ }^{9}$ Malignant gliomas often extend well beyond tumor margin as defined by the surgeons. The local recurrence and neurological impairment are frequent even after apparently complete removal of the tumor, which increases the risk of surgical resection of malignant gliomas. ${ }^{10}$ Furthermore, the nonselective toxicity of antineoplastic agents, clinical chemotherapy, and radiotherapy is also known to be toxic to the nervous system. ${ }^{11,12}$ Therefore, nerve repair in patients with glioblastoma should gain enough attention in clinical treatment.

Gangliosides consist of a hydrophilic sialic acid terminal sugar and a hydrophobic ceramide moiety, widely exist in neural tissue of vertebrates, and have been reported to modulate ion transport, neuronal differentiation, $G$ protein-coupled receptors, immune system reactivities, and neuroprotective signaling. ${ }^{13,14}$ Monosialogangliosides GM1 play an important role in regulating $\mathrm{Ca}^{2+}$ in the nucleus and maintaining neuronal homeostasis, which can affect neural function by modulating cell signaling, such as ERK1/2 phosphorylation and $\mathrm{Na}^{+} / \mathrm{Ca}^{2+}$ exchanger. ${ }^{15-17}$ Many studies have shown that GM1 gangliosides can play neuroprotective and neurorestorative roles in neuronal injury, as well as induce neuritogenesis and promote cell differentiation. ${ }^{18-20}$ More importantly, GM1 can overcome the BBB and treat neurological disease. ${ }^{21}$ Exogenously administered GM1 have been widely used in clinical treatment of neurodegenerative diseases such as Parkinson and Alzheimer. ${ }^{17,22}$

Doxorubicin (DOX) is an anthracycline antibiotic widely used in treating various types of cancers. Unfortunately, DOX is not sufficiently effective due to its poor permeability of crossing BBB to achieve the therapeutic levels. ${ }^{23}$ Moreover, the fluorescent properties of DOX provide opportunities to track the molecules and DOX-loaded nanocarriers in cells or animals by using fluorescence-based techniques without any other fluorescence labels. ${ }^{24}$ Therefore in this study, DOX was used as a model drug.

In this work, we show that amphiphilic GM1 can spontaneously self-assemble into micelles that could be used to solubilize hydrophobic drugs (DOX) in aqueous solution. The primary goal of our study is to evaluate the ability of GM1 micelles to overcome BBB and establish a novel strategy for targeting malignant brain tumors, for repairing nerves and killing tumor cells simultaneously.

\section{Materials and methods \\ Materials}

DOX was purchased from meilunepharma (Dalian, People's Republic of China). Purified monosialogangliosides GM1 were kindly provided by Huan Rui (Chongqing, People's Republic of China). DiR iodide (1,1'-dioctadecyl-3,3,3',3'tetramethyl indotricarbocyanine iodide) (DiR) fluorescent probes were purchased from KeyGen Biotech (Nanjin, People's Republic of China). Dulbecco's Modified Eagle's Medium (DMEM) and Medium 199 were purchased from HyClone (Logan, UT, USA). Trypsin (0.25\%) was purchased from Thermo Fisher Scientific (Waltham, MA, USA). 4',6-Diamidino-2-phenylindole (DAPI) was supplied by Beyotime Biotechnology (Shanghai, People's Republic of China). All other solvents used were of analytical grade.

\section{Preparation and characterization of DOX/GMI micelles}

A method was developed and validated for preparing mixed micelles of GM1 and DOX according to Leonhard et al. ${ }^{25}$ A stock solution of purified monosialogangliosides GM1 was prepared in triple-distilled water $24 \mathrm{~h}$ prior to use. The solution was maintained at $4{ }^{\circ} \mathrm{C}$ for $24 \mathrm{~h}$, and then centrifuged at $10,000 \times g$ for $15 \mathrm{~min}$, and the supernatant was filtered through $0.22-\mu \mathrm{m}$ filter.

A stock solution of DOX was prepared in dimethyl sulfoxide (DMSO). Then, the DOX solution was slowly added to GM1 micelles solution.

The DOX-loaded GM1 micelles were incubated at $4{ }^{\circ} \mathrm{C}$ for $24 \mathrm{~h}$ and dialyzed for $24 \mathrm{~h}$ to remove all the DMSO.

\section{Optimizing preparation of DOX/GMI micelles}

The concentration and molar ratio of both drug and carrier are considered as key factors in the preparation of micelles. Orthogonal experimental design (OED) method was used for constructing the best combination levels for different factors (Table 1). GM1 concentration (A), DOX concentration (B), and GM1/DOX molar ratio $(\mathrm{C}: \mathrm{A} \times \mathrm{B})$ varied at three different levels when preparing micelles. The drug-loading efficiency of these nine combinations was evaluated by software SPSS to identify the best combination. 
Table I Factors and levels of the preparation of DOX/GMI micelles

\begin{tabular}{|c|c|c|c|}
\hline \multirow[t]{2}{*}{ Levels } & \multicolumn{3}{|l|}{ Factors } \\
\hline & $\begin{array}{l}\text { A: GMI } \\
\text { concentration } \\
\left(\mathbf{m g} \cdot \mathbf{m L}^{-1}\right)\end{array}$ & $\begin{array}{l}\text { B: DOX } \\
\text { concentration } \\
\left(\mathbf{m g} \cdot \mathbf{m L}^{-1}\right)\end{array}$ & $\begin{array}{l}\text { C: GMI/DOX } \\
(\mathrm{mol} / \mathrm{mol})\end{array}$ \\
\hline LI & 100 & 6 & $50 / 1$ \\
\hline L2 & 50 & 3 & $25 / 1$ \\
\hline L3 & 25 & 1.5 & $5 / 1$ \\
\hline
\end{tabular}

Abbreviation: DOX, doxorubicin.

\section{Size and surface charge determination}

Micelles size and zeta potential were measured by dynamic light scattering (DLS) using Zetasizer (Nano ZS90; Malvern Instruments, Malvern, UK) at $25^{\circ} \mathrm{C}$ and refractive index of 1.57 .

\section{Electron microscopy}

The morphology of the micelles was characterized through a JEM 1200EX transmission electron microscope. The samples were prepared by loading $30 \mu \mathrm{L}$ on the carboncoated copper grid, which was allowed to stand for $5 \mathrm{~min}$, and the redundant solution was absorbed with filter paper. The copper grid was dyed with a drop of uranyl acetate for $90 \mathrm{~s}$ and then dehydrated.

\section{DOX loading and encapsulation}

Ultraviolet spectroscopy was followed to evaluate the drug loading of DOX/GM1 micelles on a UV spectrophotometer (T6-New Century; Purkinje General Instrument Co., Ltd. Beijing, People's Republic of China). Lyophilized DOXloaded micelles were dissolved in N,N-dimethylformamide (DMF) and analyzed by measuring the absorbance, wherein calibration curve of DOX/DMF was established at $480 \mathrm{~nm}$ with enhanced concentrations of DOX in the range of 10-45 $\mathrm{mg} \cdot \mathrm{mL}^{-1}$. The drug loading and encapsulation efficiency were calculated as follows:

Loading efficiency $\%=\frac{\text { Drug contents of mixed micelles }}{\text { The weight of mixed micelles }}$

$\begin{aligned} & \text { Encapsulation } \\ & \text { efficiency } \%\end{aligned}=\frac{\text { Drug content of mixed micelles }}{\text { The quantity of reagent }}$.

\section{DOX release in vitro}

We assessed the drug release profile of DOX/GM1 in phosphate-buffered saline (PBS, $0.01 \mathrm{M}, \mathrm{pH}$ 7.4) through a dialysis bag of molar weight cutoff $(\mathrm{MWCO})=2,000$. The calibration curve of DOX in PBS buffer was established at
$480 \mathrm{~nm}$, and a linear relationship was obtained with a correlation coefficient of 0.9996 . Lyophilized micelles $(20 \mathrm{mg})$ were dissolved in $2 \mathrm{~mL}$ PBS, and the solution was then transferred to dialysis bags which were immersed in PBS buffer. The dissolution medium $(20 \mathrm{~mL})$ was placed in an incubator at $37^{\circ} \mathrm{C}$ and $100 \mathrm{rpm}$ throughout the drug release experiment. At selected time intervals, 3-mL sample was collected with the replacement of the same volume of fresh PBS solution. The release amount of DOX was evaluated using ultraviolet (UV) detection.

\section{Cell culture}

Human umbilical vein endothelial cells (HUVECs) (China Center for Type Culture Collection) were cultured in the endothelial cell culture medium (high-glucose DMEM, 10\% FBS, $100 \mathrm{U} / \mathrm{mL}$ penicillin, $100 \mu \mathrm{g} \cdot \mathrm{mL}^{-1}$ streptomycin) and digested by $0.25 \%$ trypsin for about $30 \mathrm{~s}$.

C6 glioma cells (Kunming Cell Bank, CAS, Kunming, People's Republic of China) were grown in RPMI 1640 medium supplemented with $2.5 \%(\mathrm{v} / \mathrm{v}) \mathrm{FBS}, 10 \%(\mathrm{v} / \mathrm{v})$ horse serum, $100 \mathrm{U} / \mathrm{mL}$ penicillin, and $100 \mu \mathrm{g} \cdot \mathrm{mL}^{-1}$ streptomycin, and digested by $0.05 \%$ trypsin (diluted with PBS solution) for about $20 \mathrm{~s}$.

Cells were maintained in a $5 \% \mathrm{CO}_{2}$ incubator at $37^{\circ} \mathrm{C}$.

\section{In vitro cellular uptake}

Cellular uptake of micelles was studied by confocal laser scanning microscopy (CLSM, Leica TCS SP5). HUVECs and $\mathrm{C} 6$ cells were seeded in laser confocal petri dishes at a density of $2 \times 10^{5}$ cells per dish and grown for $24 \mathrm{~h}$ with $5 \% \mathrm{CO}_{2}$ at $37^{\circ} \mathrm{C}$. The cells were incubated with DOX $\left(10 \mu \mathrm{g} \cdot \mathrm{mL}^{-1}\right)$ solution and DOX/GM1 micelles for $0.5,2$, and 4 , respectively. The cells were washed with PBS ( $\mathrm{pH} 7.4$ ) for three times and fixed with 4\% paraformaldehyde for $15 \mathrm{~min}$. The liquid was removed and washed with PBS twice. DAPI was added to stain nuclei. Then, cells were observed by CLSM.

\section{In vivo distribution behavior of DiR-loaded micelles}

The Institutional Animal Care and Use Committee of Chongqing University approved all animal (including mice and zebrafish) experiments, procedures, and protocols, and all animals were cared for in accordance with the guide for the Care and Use of Laboratory Animals.

The male Kunming (KM) mice, with body weights of 20-23 g, were procured from Laboratory Animal Center, Third Military Medicinal University (Chongqing, People's Republic of China). The preparation of DiR-loaded micelles 
is consistent with the method of preparation of mixed micelles of GM1 and DOX. Mice were starved for $12 \mathrm{~h}$ before receiving a single dose ( $25 \mu \mathrm{g} /$ mouse $)$ of the DiR and DiR/GM1 micelles intravenously. Mice were monitored by In-Vivo FX PRO for small-animal imaging after anesthesia.

\section{Transgenic fluorescent zebrafish for micelles to overcome BBB}

Transgenic fluorescent zebrafish (flk1: GFP) was incubated at $28^{\circ} \mathrm{C}$ according to standard zebrafish care and procedures. Four days postfertilization (dpf), zebrafish embryos were anesthetized with tracaine $\left(4 \mathrm{~g} \cdot \mathrm{L}^{-1}\right)$. Intracardiac injection with 5-nL DOX or DOX/GM1 using a PV 820 Pneumatic PicoPump was followed by fixing with $1.2 \%$ low-melting point agarose. Finally, the embryos were imaged at different time points (20 min, $60 \mathrm{~min}$ ) using CLSM.

\section{Therapeutic efficacy in tumor-bearing rats}

Male Wistar rats weighing $300 \pm 10 \mathrm{~g}$ were purchased from Chongqing Academy of Chinese Materia Medica (Chongqing, People's Republic of China). All animals were kept under standard conditions with free access to food and water. The cranium was exposed after animals were deeply anesthetized with $10 \%$ chloralic hydras. Approximate $5 \times 10^{5}$ C6 glioma cells/15 $\mu \mathrm{L}$ in 1640 medium were stereotaxically implanted into the right forebrain of each rat at the specific site: $1.0 \mathrm{~mm}$ anterior and $3.0 \mathrm{~mm}$ lateral from the bregma at a depth of $4.5 \mathrm{~mm}$ from the brain surface. ${ }^{26}$ Eight days after tumor inoculation, rats were randomly divided into five groups $(\mathrm{n}=5)$. The DOX solution, GM1 solution, DOX + GM1 and DOX/GM1 micelles, and physiological saline (blank control group) were injected intravenously via tail vein with DOX concentration of $3 \mathrm{mg} / \mathrm{kg}$ per dose, and all the solutions except DOX and saline had the same GM1 concentration. The drugs were given every 3 days with a total of three doses per rat. On day 20, one rat from each group was monitored by magnetic resonance imaging (MRI) for measuring the tumor size, and the animals were sacrificed with brain tissue dissected for hematoxylin and eosin (H\&E) staining. The survival outcome and neurological examination were calculated daily after inoculation of C6 tumor cells. Kaplan-Meier survival curves were plotted for each group.

\section{Results and discussion}

Preparation and characterization of DOX/GMI micelles

Gangliosides are sialic acid-containing glycosphingolipids and generated mostly in the nervous system. ${ }^{27,28}$ In aqueous solution, most gangliosides form micelles with very low critical micelle concentration values, of the order of $10^{-10}-10^{-8} \mathrm{M}^{29,30}$ These properties suggest that a novel GM1 micelles system can be constructed for hydrophobic or amphipathic active pharmaceuticals. Both molar ratio of GM1/DOX and their concentrations could play important roles in the formation of DOX-loaded GM1 micelles. The optimum micelles (GM1 concentration: $50 \mathrm{mg} \cdot \mathrm{mL}^{-1}$, DOX concentration: $3 \mathrm{mg} \cdot \mathrm{mL}^{-1}$, GM1/DOX: 5/1) were obtained through the OED method.

The DOX/GM1 micelles were prepared using dialysis method. The DLS analysis revealed that the DOX-loaded GM1 aggregated to particles with an average size of $226 \mathrm{~nm}$ (Figure 1A). The zeta potential measurement showed that surface was negatively charged with $-45.7 \mathrm{mV}$ (Figure 1B). The high magnitude of zeta potential can prevent particle aggregation during storage. ${ }^{31}$ Compared to cationic NPs, anionic NPs cause less disruption of plasma-membrane integrity, weaker damage of lysosomal and mitochondrial membrane, ${ }^{32}$ and less neuron toxic response. ${ }^{33}$ As shown in transmission electron microscopy (TEM) images, the DOX-loaded GM1 micelles were spherical with an average diameter of 5-21 nm, but the particles size was mainly between 8 and $14 \mathrm{~nm}$ for the mixed micelles (Figure 1C). However, the particle size measured by TEM was smaller than that measured by DLS. This is mainly because DLS measures aggregate size in the hydrated state, while the particle is dehydrated when measured by TEM. ${ }^{34}$

Due to its amphiphilicity, GM1 can spontaneously selfassemble into micelles which enables solubilization of hydrophobic DOX in an aqueous environment. DOX was loaded onto GM1 micelles by dialysis method. The concentration of DOX/GM1 micelle was $9.33 \%$, and the micelle showed high encapsulation efficiency $(97.05 \%)$.

The in vitro drug release experiments were carried out for DOX/GM1 micelles with an NP concentration of $10 \mathrm{mg} \cdot \mathrm{mL}^{-1}$, and DOX release profiles were studied under physiological condition ( $\left.\mathrm{pH} 7.4,37^{\circ} \mathrm{C}\right)$. The results demonstrated that the release of DOX from micelles was slow and sustainable without a burst release effect (Figure 1D). The cumulative release of DOX was limited to $66.53 \%$ over a period up to 16 days. The release rate of DOX decreased gradually. It was found that $45.75 \%$ of drug was released within the first $51 \mathrm{~h}$. The releasing quantity after $51 \mathrm{~h}$ was about $20 \%$. It is generally recognized that the physical process of encapsulation of hydrophobic drugs in GM1 micelle is mainly driven by the hydrophobic interaction between drug and hydrophobic segments of ganglioside. ${ }^{35}$ The interactions between hydrophobic portion and DOX, and slow degradation of ganglioside may be responsible for the controlled drug 
A

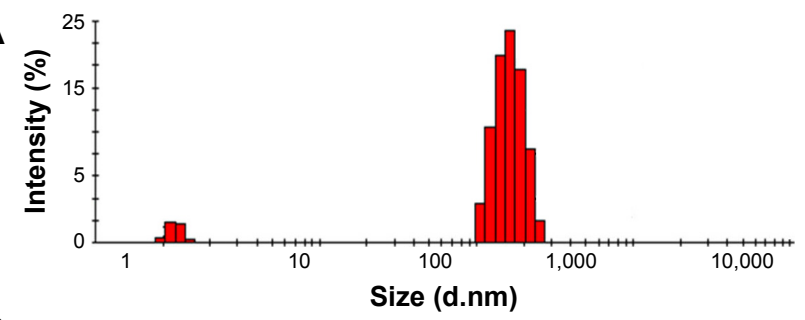

B
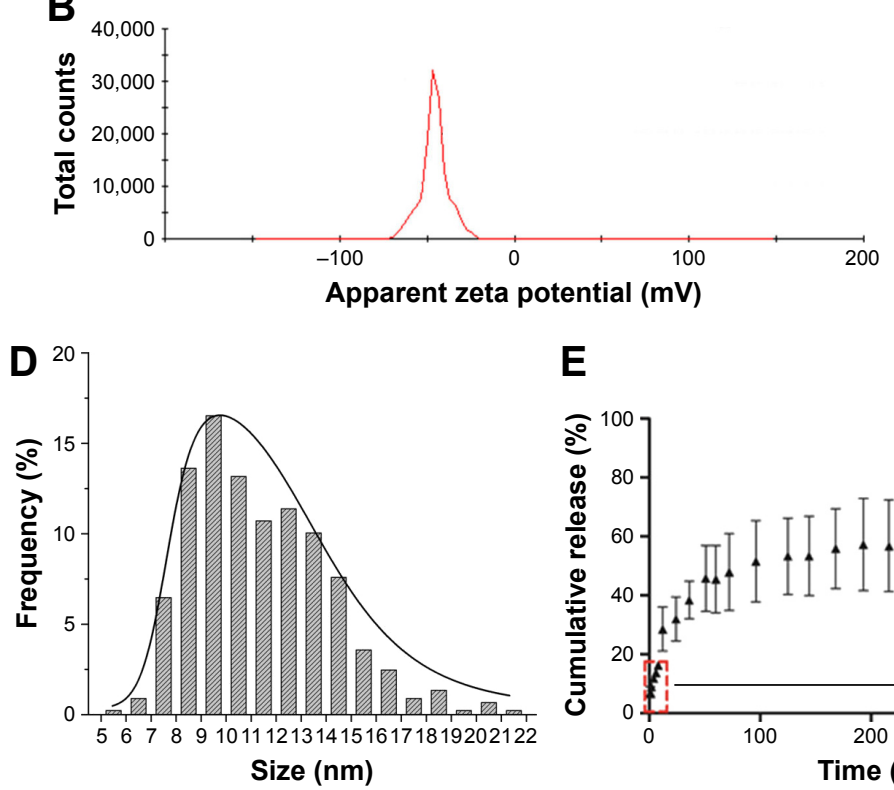

C

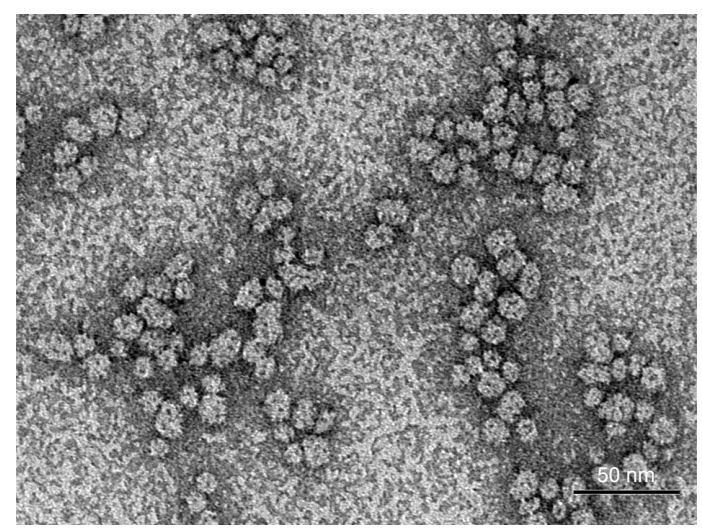

E

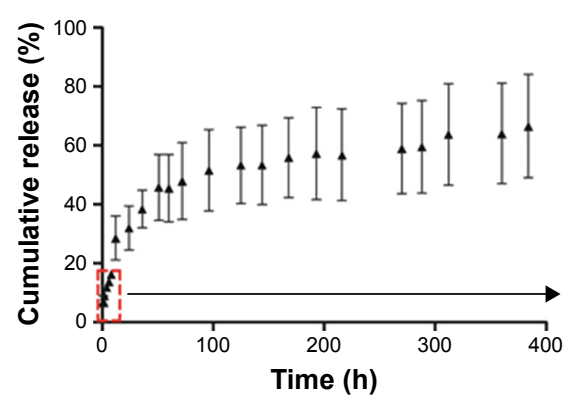

$\mathbf{F}$

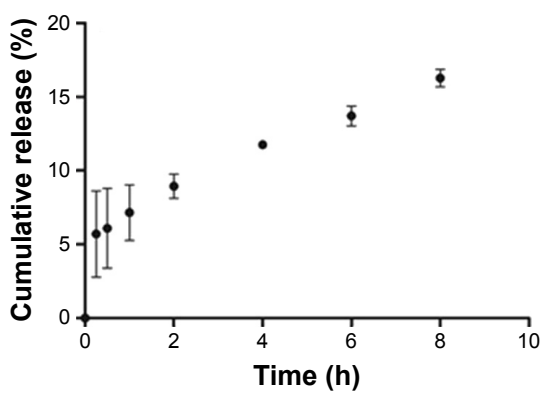

Figure I Size distribution (A) and surface potential (B) were determined by dynamic light scattering. Transmission electron microscopy (C) and frequency histograms (D) of DOX-loaded GMI micelles. Scale bar: $50 \mathrm{~nm}$. Drug release profile of DOX from GMI micelles at pH 7.4 and $37^{\circ} \mathrm{C}$ in PBS buffer (E and $\left.\mathbf{F}\right)$.

Abbreviations: DOX, doxorubicin; PBS, phosphate-buffered saline.

release. As stated above, DOX-loaded micelle seems to be a promising and viable technology platform for controlled drug delivery.

\section{In vitro cellular uptake study}

The cellular uptake efficiency of micelles was studied in HUVECs and C6 cells through CLSM with DAPI staining. ${ }^{36}$ As shown in Figure 2, HUVECs and C6 cells treatment with free DOX displayed DOX fluorescence in cytoplasm after $0.5 \mathrm{~h}$, and the fluorescence was distributed in nucleus within a short period of 2-4 h. In comparison, the red fluorescence was observed after $0.5 \mathrm{~h}$ and enhanced in nuclei after incubation with DOX/GM1. There was no significant difference in fluorescence intensity between $2-\mathrm{h}$ and $4-\mathrm{h}$ incubation. Scanning electron microscopy results indicated that free DOX entered the cell and gathered at nucleus but was excreted with reduced nuclear fluorescence. Removal of DOX from the nucleus is likely mediated by P-glycoprotein located in the plasma membrane, Golgi apparatus, and nucleus, which serves as an ATP-coupled active drug efflux pump of hydrophobic compounds conferring multidrug resistance (MDR) to HUVECs and C6 cells. ${ }^{37}$ However, it is a notable finding that in this study, the efflux mechanism failed to pump out DOX/GM1 micelles. The use of targeted micelles for chemotherapeutic delivery may conceivably circumvent the MDR drug efflux mechanism, which leads to resistance, albeit the mechanism is unclear. ${ }^{38}$ In recent years, numerous carrier-mediated transporters or receptors have demonstrated that BBB has the capability to control the uptake of chemotherapeutics into the brain. Drug delivery systems (NPs, micelles, etc.) have been used to strengthen the ability of overcoming the $B B B$ via the binding effect between specific ligands equipped on the surface of the delivery system and the specific endogenous transporters localized on the BBB. ${ }^{39}$ The uptake of DOX/GM1 micelles may be attributed to that polymersome nanocarriers functionalized with GM1-targeting peptides could bind $\mathrm{BBB}$ receptors and get transferred in the brain endothelial cells in vitro. ${ }^{40}$

\section{In vivo distribution behavior of DiR-loaded micelles}

The In-Vivo FX PRO animal imaging system was used for investigating the in vivo biodistribution and brain-targeting efficiency of DiR/GM1 micelles. As shown in Figure 3, 


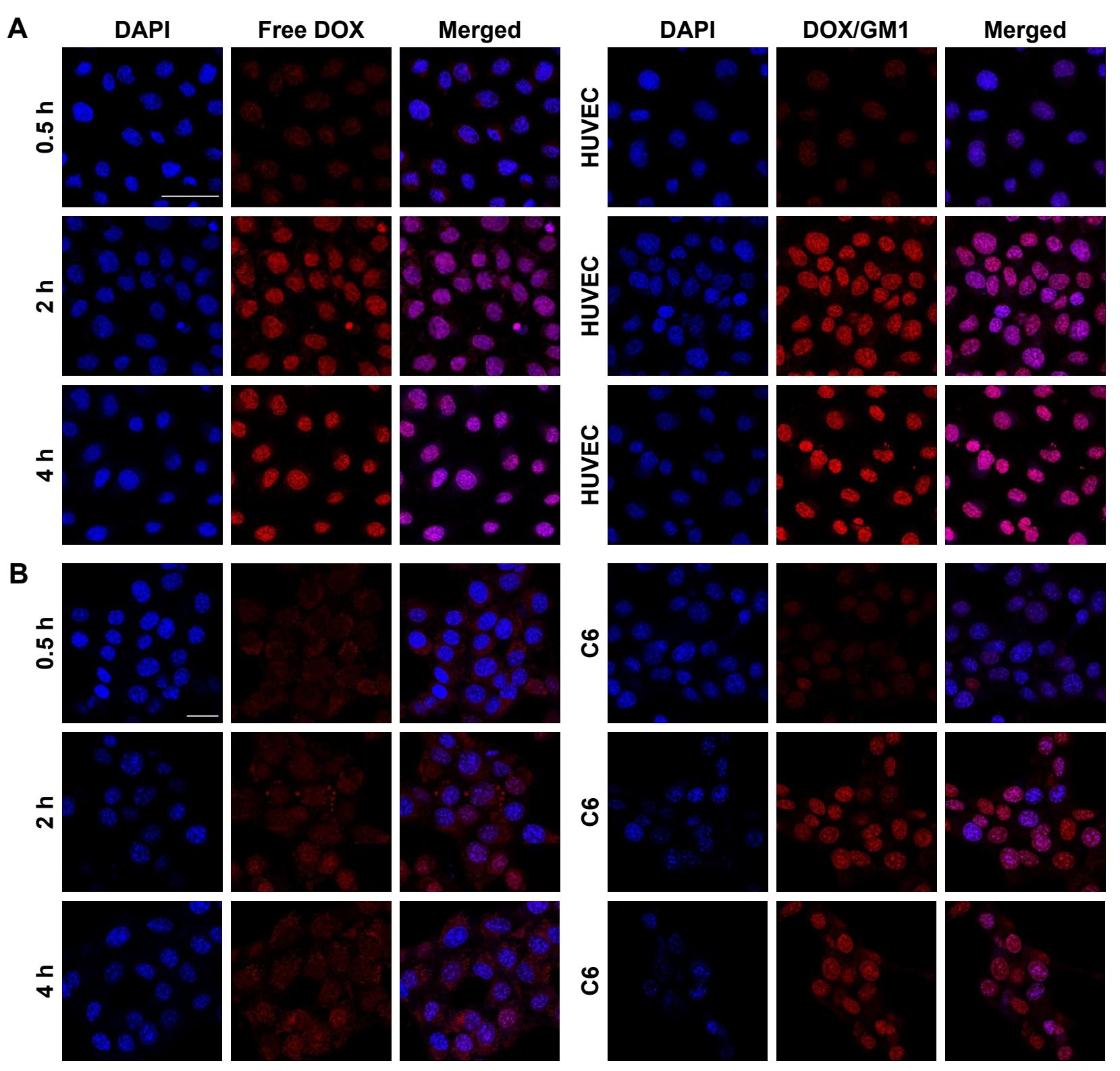

Figure 2 Kinetics of cellular uptake of free DOX and DOX/GMI incubated with HUVECs (A, scale bars: $50 \mu \mathrm{m}$ ) and C6 cells (B, scale bars: $25 \mu \mathrm{m}$ ), respectively. Abbreviations: DAPI, 4',6-diamidino-2-phenylindole; DOX, doxorubicin; HUVECs, human umbilical vein endothelial cells.

fluorescence signal of control group was observed in the liver after free DOX injection, due to the cellular uptake by phagocytic cells in reticuloendothelial system. ${ }^{41}$ Moreover, in DiR-loaded GM1 micelles, fluorescence signal from $\mathrm{DiR} / \mathrm{DOX}$ micelles was observed in the mice brain during the whole monitoring period. The fluorescence signal intensity was strong until $9 \mathrm{~h}$ and became weak after $24 \mathrm{~h}$. As a result of the physical formation process, GM1 micelles may still maintain the same specific groups with GM1 molecule, which contributes to transmembrane transportation. As demonstrated in a previous study, ${ }^{25}$ when interacting with blood, the GM1/Ptx mixed micelles form ternary complexes with albumin, and albumin binding to the gp60 receptor of endothelial cells could promote transcytosis. In addition, GM1 ganglioside facilitates long circulating time in vivo by suppressing phagocytosis. ${ }^{42}$

\section{Transgenic fluorescent zebrafish for micelles to overcome BBB}

To study the ability of DOX-loaded GM1 micelle to cross the BBB in vivo, DOX/GM1 micelle and DOX solution were injected into the circulation of zebrafish (4 dpf) through the heart, and the in vivo imaging of the brain was monitored. As expected, mixed micelles extravasated extensively from 

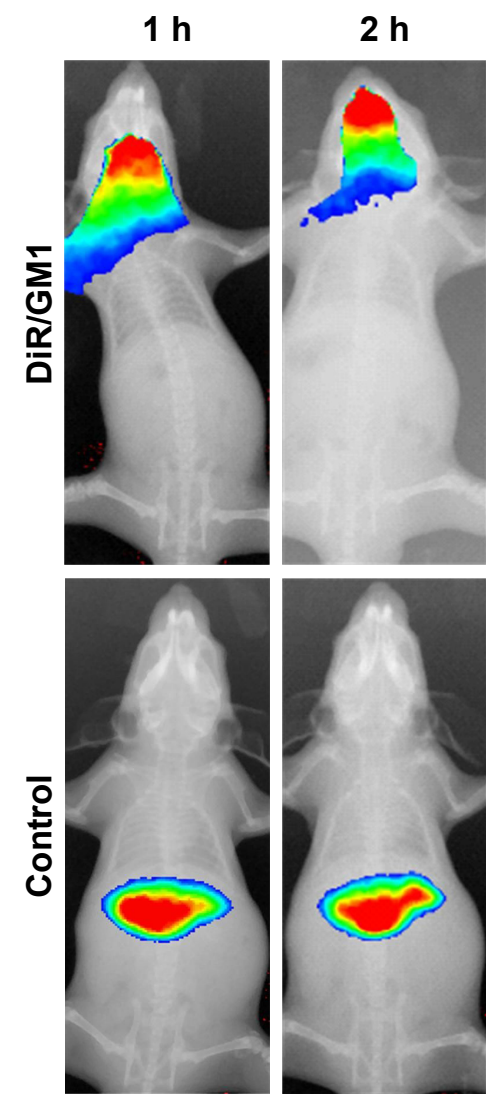

$2 \mathrm{~h}$

$4 \mathrm{~h}$
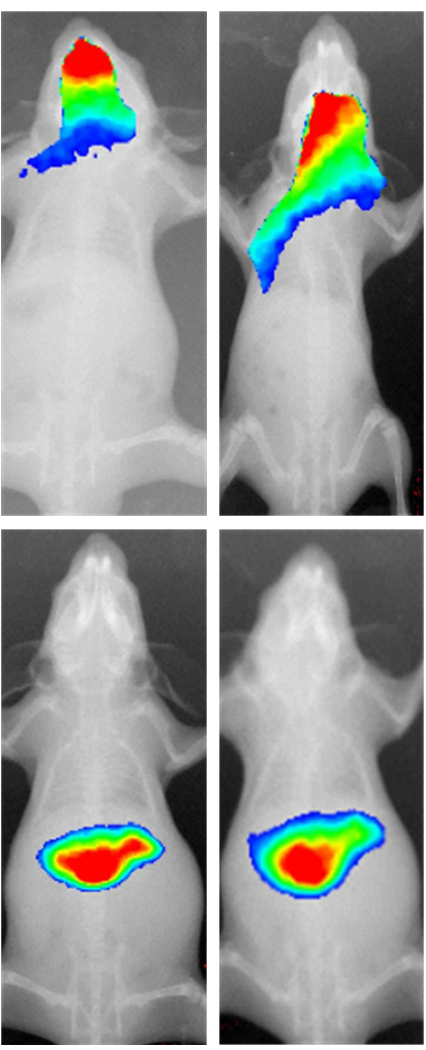

$6 \mathrm{~h}$
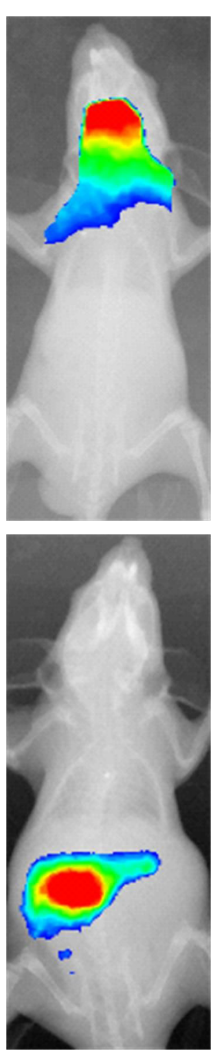

$9 \mathrm{~h}$
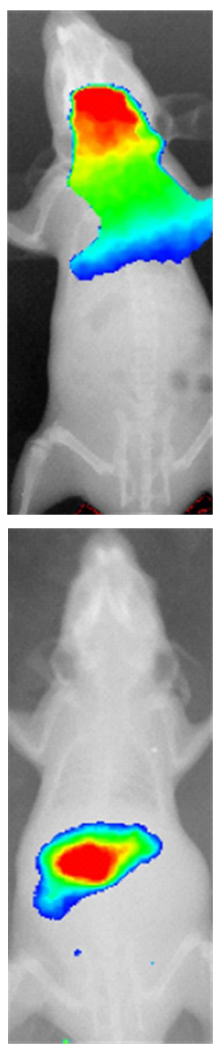

$24 \mathrm{~h}$
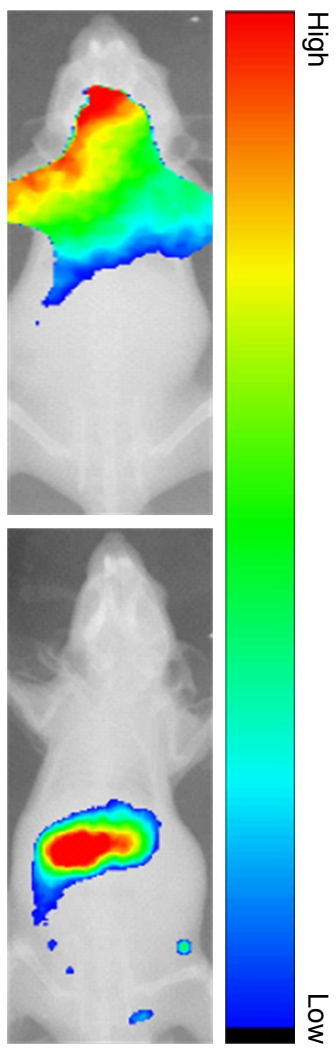

Figure 3 In vivo fluorescence imaging of Kunming mice at different time points after administration with free DiR (control) and DiR/GMI micelle. Abbreviation: DiR, I,I'-dioctadecyl-3,3,3',3'-tetramethyl indotricarbocyanine iodide.

vessels (green), as evidenced by fluorescence of DOX (red) within the brain parenchyma (Figure 4, arrows). In contrast, DOX was not observed in the brain but confined to the blood vessels. The results indicated that GM1 micelles could effectively deliver the drugs into brain tissue by overcoming the BBB, although astroglial structures in zebrafish brain differ from mammalian astrocytes. ${ }^{43}$ Due to rapid growth, large number of offspring, and a similar BBB function to human, ${ }^{44}$ zebrafish is suitable for the study of BBB and drug delivery. Only 3 days after fertilization, embryonic zebrafish shows the development of a functional endothelial-based BBB that has similar properties to mammals, ${ }^{45}$ and it is used in many studies focusing on overcoming BBB. ${ }^{46,47}$ Furthermore, the BBBcrossing ability of DOX/GM1 micelles could be observed intuitively in the transgenic fluorescent zebrafish (flk1: GFP) due to its vascular labeling. No studies have suggested that GM1 micelle is a viable carrier for delivering drugs across the BBB. However, our results demonstrated that GM1 micelles facilitated DOX delivery to the brain. In the experiment with zebrafish, no fluorescence was detected in the group injected with DOX, while there was significant penetration of the $\mathrm{BBB}$ as demonstrated by the red fluorescence in DOX/GM1 micelles. Therefore, the results of biodistribution assays and BBB penetration in vivo indicated the feasibility of using GM1 ganglioside micelle as a brain carrier.

\section{Therapeutic efficacy in tumor-bearing rats}

To reveal the potential antitumor and nerve injury-repairing functions of DOX/GM1 in glioma therapy, brain tumorbearing rat models were established and treated with different GM1 and DOX formulations via tail intravenous injection.

Twenty days after $\mathrm{C} 6$ implantation, one rat in each group was randomly selected to monitor the tumor growth through MRI (MAGNETOM Avanto 1.5T). $\mathrm{T}_{2}$ weighted images are shown in Figure 5A (MRI); the obvious tumor was found in all groups. The size of the tumor in control group was up to $0.9 \times 0.8 \mathrm{~cm}$, whereas the size was $0.64 \times 0.58,0.68 \times 0.61$, $0.66 \times 0.43$, and $0.4 \times 0.4 \mathrm{~cm}$ in GM1, DOX, GM1 + DOX, and DOX/GM1 groups, respectively. The smallest tumor size in DOX/GM1 group demonstrated that it has the best antitumor capacity compared to other treatment groups.

The histopathological changes in glioma of all treatment groups were examined 20 days after tumor implantation. 


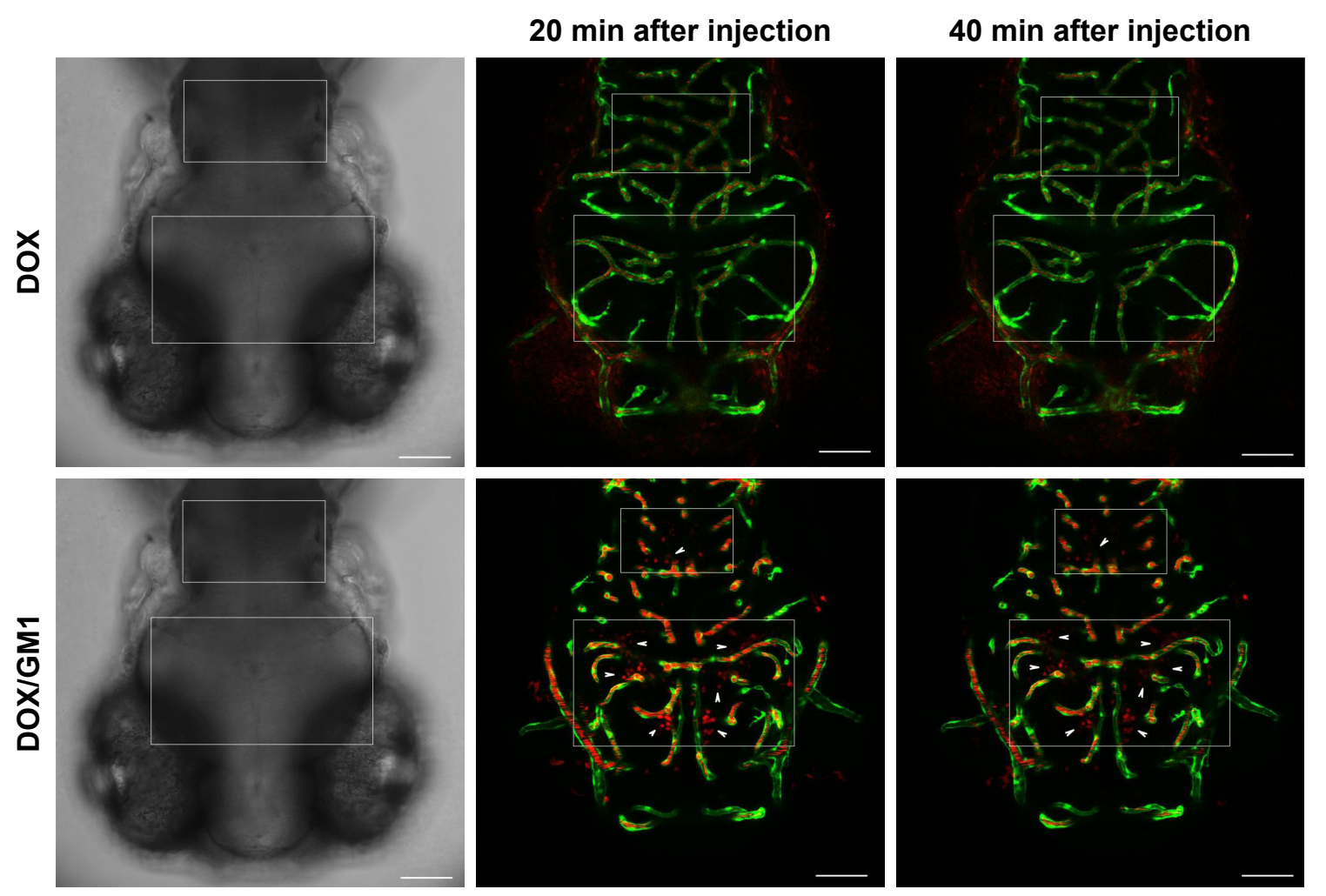

Figure 4 Intracardiac injection of DOX/GMI micelle and DOX into transgenic (flkl: GFP) zebrafish. The arrowhead indicates micelles crossing out of vessels (green). DOX retained within vessels without the GMI micelle is shown in red. Scale bars: $75 \mu \mathrm{m}$. Abbreviation: DOX, doxorubicin.

The H\&E staining (Figure 5B) was performed to evaluate tumor necrosis. ${ }^{48}$ Negligible necrosis of tumor was observed in the group treated with saline, while the group treated with GM1 + DOX exhibited obvious necrosis which was more significant than GM1 or DOX group. Furthermore, much more extensive necrosis phenomenon was detected in glioma tissues treated with DOX/GM1 micelles. These H\&E staining results also indicated the therapeutic effects of these treatment groups, which were consistent with results of MRI.

Moreover, to assess the antitumor effects of different treatment groups, the survival time of tumor-bearing rats is presented as a Kaplan-Meier plot in Figure 5C. The survival time of rats in the saline control group was 17-23 days (median survival: 19.6 days). The median survival time was 21.6, 23.4, 24.8, and 26.8 days for GM1, DOX, DOX + GM1, and DOX/GM1 groups, respectively. Animals treated with GM1 solution had a little difference in survival time compared to saline group; the possible reason was that GM1 could repair nerve injuries caused by tumor growth. Though DOX has poor targeting efficiency, DOX solution could prolong the median survival time, which is mainly ascribed to its broad-spectrum anticancer activity. ${ }^{49}$ DOX + GM1 solution combined the advantages of antitumor effect and neuron repair, resulting in longer median survival time. Because of $\mathrm{BBB}$ penetration, the DOX/GM1 group showed prolonged survival among all the treatment groups.

In our studies, the neurological function of rats was recorded after implantation of C6 tumor cells to evaluate the neuroprotective and neurorestorative roles of GM1 in neuronal injury. The assessment of neurological score was in accordance with the Garcia scale, including spontaneous activity, symmetry in the movement of four limbs, forepaw outstretching, climbing, body proprioception, and vibrissae touch, which is scored on a scale from 3 to $18 .^{50}$ As shown in Figure 5D, the neuroscore of rats in GM1, GM1 + DOX, and GM1/DOX groups remained as at least 10 points, while the conditions of the rats treated with saline and DOX solution were not satisfactory, which showed significant neurological impairment before the rats' death. The results were consistent with previous studies which showed GM1 ganglioside promotes functional recovery of the damaged nerves, and also confirmed GM1 micelle is capable of passing the BBB.

In summary, the therapeutic effect of DOX/GM1 micelles was more powerful than normal saline, free DOX, free GM1, 

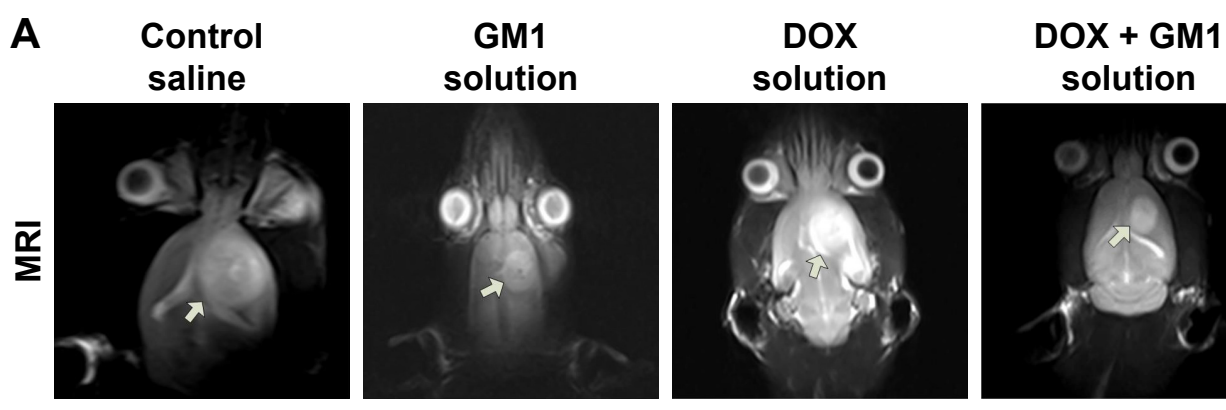

solution
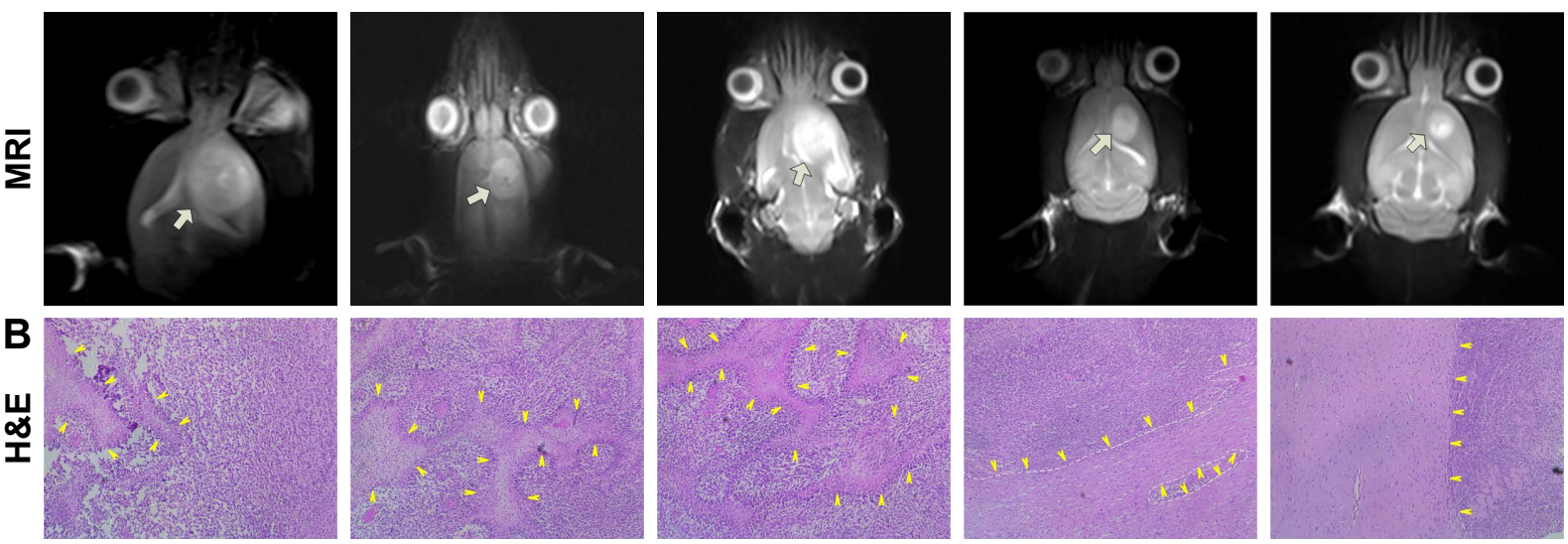
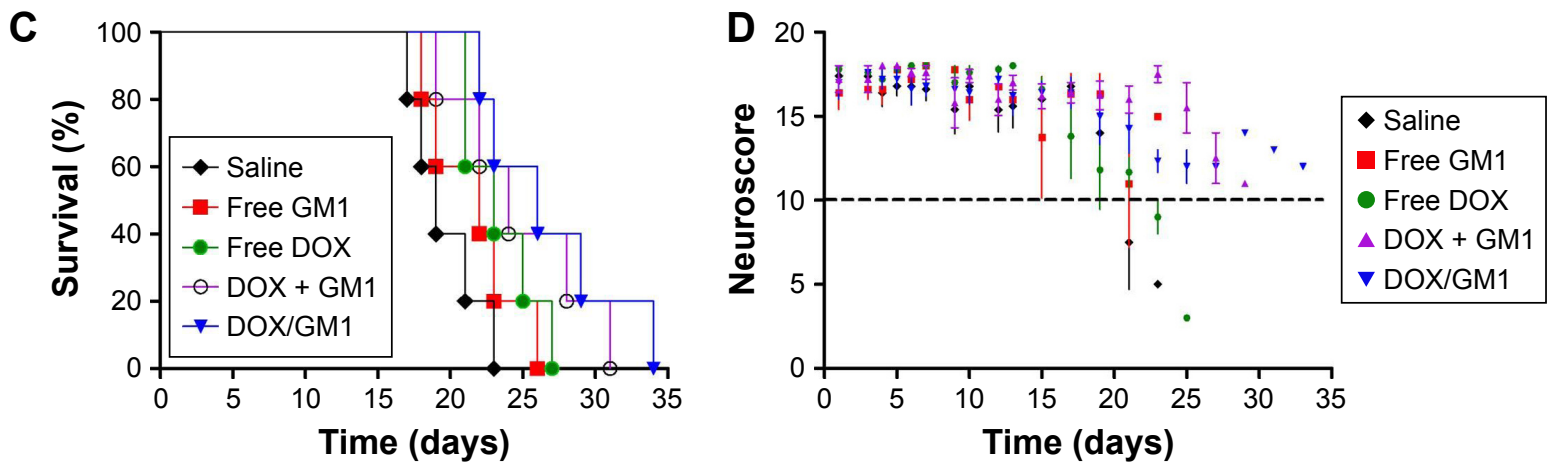

Figure 5 MRI images (A), H\&E staining (B, $\times 100)$, Kaplan-Meier plot (C), and neuroscore (D) of tumor-bearing rats after treatment with saline, free GMI solution, free DOX solution, DOX + GMI solution, and DOX/GMI micelle, respectively. White arrows in MRI images indicate the tumor, and yellow arrows in H\&E staining indicate tumor necrosis.

Abbreviations: DOX, doxorubicin; H\&E, hematoxylin and eosin.

and DOX + GM1, as demonstrated by prolonged survival time, reduced tumor size, and extensive tumor necrosis by histological examination. More importantly, our data confirmed that GM1 formulations could improve nerve functions of the animals with brain tumors.

\section{Conclusion}

In this study, ganglioside GM1 micelles were designed and synthesized. The anticancer drug DOX was spontaneously encapsulated into the nanomicelles. In aqueous solution, the mixed micelles self-assembled into spherical and uniform NPs which exhibited slow drug release. In addition, the DOX/GM1 micelles showed promising BBB-overcoming and brain-targeting efficacy in vitro and in vivo. As stated above, GM1 micelles, a novel nanodelivery system, can not only penetrate BBB effectively but also repair nerves at the same time. Thus, the drug-loaded GM1 micelles with multiple functions have great potential for drug delivery to treat intracranial diseases such as brain tumor and neurodegenerative diseases.

\section{Acknowledgments}

This work was supported by the National Key Basic Research Project (973) (2014CB541600), the Fundamental Research Funds for the Central Universities (2015CDJZR), graduate scientific research and innovation foundation of Chongqing, People's Republic of China (Grant No CYS16015), and National Natural Science Foundation of China (31200713). In particular, the authors would like to acknowledge Mr Fei Fang (College of Bioengineering, Chongqing University) for his help in collecting data on BBB-overcoming potential of micelles using transgenic fluorescent zebrafish.

\section{Disclosure}

The authors report no conflicts of interest in this work. 


\section{References}

1. Cloughesy TF, Cavenee WK, Mischel PS. Glioblastoma: from molecular pathology to targeted treatment. Annu Rev Pathol. 2014;9:1-25.

2. Chen J, McKay RM, Parada LF. Malignant glioma: lessons from genomics, mouse models, and stem cells. Cell. 2012;149(1):36-47.

3. Silva AC, Oliveira TR, Mamani JB, et al. Application of hyperthermia induced by superparamagnetic iron oxide nanoparticles in glioma treatment. Int J Nanomedicine. 2011;6:591-603.

4. Stupp R, Hegi ME, Gorlia T, et al; European Organisation for Research and Treatment of Cancer (EORTC); Canadian Brain Tumor Consortium; CENTRIC study team. Cilengitide combined with standard treatment for patients with newly diagnosed glioblastoma with methylated MGMT promoter (CENTRIC EORTC 26071-22072 study): a multicentre, randomised, open-label, phase 3 trial. Lancet Oncol. 2014;15(10):1100-1108.

5. Pardridge WM. Blood-brain barrier delivery. Drug Discov Today. 2007; 12(1-2):54-61.

6. Li Y, He H, Jia X, Lu WL, Lou J, Wei Y. A dual-targeting nanocarrier based on poly (amidoamine) dendrimers conjugated with transferrin and tamoxifen for treating brain gliomas. Biomaterials. 2012;33(15):3899-3908.

7. Caraglia M, De Rosa G, Salzano G, et al. Nanotech revolution for the anti-cancer drug delivery through blood-brain-barrier. Curr Cancer Drug Targets. 2012;12(3):186-196.

8. De Rosa G, Salzano G, Caraglia M, Abbruzzese A. Nanotechnologies: a strategy to overcome blood-brain barrier. Curr Drug Metab. 2012; 13(1):61-69.

9. Onishi M, Ichikawa T, Kurozumi K, Date I. Angiogenesis and invasion in glioma. Brain Tumor Pathol. 2011;28(1):13-24.

10. Parvez T. Present trend in the primary treatment of aggressive malignant glioma: glioblastoma multiforme. Technol Cancer Res Treat. 2008; 7(3):241-248.

11. Chen XF, Wang R, Yin YM, et al. The effect of monosialotetrahex osylganglioside (GM1) in prevention of oxaliplatin induced neurotoxicity: a retrospective study. Biomed Pharmacother. 2012;66(4): 279-284.

12. Albers JW, Chaudhry V, Cavaletti G, Donehower RC. Interventions for preventing neuropathy caused by cisplatin and related compounds. Cochrane Database Syst Rev. 2014;(3):CD005228.

13. Allende ML, Proia RL. Lubricating cell signaling pathways with gangliosides. Curr Opin Struct Biol. 2002;12(5):587-592.

14. Ledeen RW, Wu G. The multi-tasked life of GM1 ganglioside, a true factotum of nature. Trends Biochem Sci. 2015;40(7):407-418.

15. Ledeen $\mathrm{R}, \mathrm{Wu} \mathrm{G}$. New findings on nuclear gangliosides: overview on metabolism and function. J Neurochem. 2011;116(5):714-720.

16. Schengrund CL. Gangliosides: glycosphingolipids essential for normal neural development and function. Trends Biochem Sci. 2015;40(7): $397-406$.

17. Wu G, Lu ZH, Kulkarni N, Amin R, Ledeen RW. Mice lacking major brain gangliosides develop parkinsonism. Neurochem Res. 2011;36(9): 1706-1714.

18. Ariga T, McDonald MP, Yu RK. Role of ganglioside metabolism in the pathogenesis of Alzheimer's disease - a review. J Lipid Res. 2008;49(6): 1157-1175.

19. Mocchetti I. Exogenous gangliosides, neuronal plasticity and repair, and the neurotrophins. Cell Mol Life Sci. 2005;62(19-20):2283-2294.

20. Skaper SD, Leon A, Toffano G. Ganglioside function in the development and repair of the nervous system. From basic science to clinical application. Mol Neurobiol. 1989;3(3):173-199.

21. Li S, Xiao N, Zhang X, et al. Effects of exogenous ganglioside-1 on learning and memory in a neonatal rat model of hypoxia-ischemia brain injury. Neural Regen Res. 2008;3(9):1004-1009.

22. Williams TL, Serpell LC. Membrane and surface interactions of Alzheimer's A $\beta$ peptide - insights into the mechanism of cytotoxicity. FEBS J. 2011;278(20):3905-3917.
23. Hanušová V, Tomšík P, Kriesfalusyová L, Pakostová A, Boušová I, Skáalová L. In vivo effect of oracin on doxorubicin reduction, biodistribution and efficacy in Ehrlich tumor bearing mice. Pharmacol Rep. 2013;65(2):445-452.

24. Blazkova I, Viet Nguyen H, Kominkova M, et al. Fullerene as a transporter for doxorubicin investigated by analytical methods and in vivo imaging. Electrophoresis. 2014;35(7):1040-1049.

25. Leonhard V, Alasino RV, Bianco ID, Garro AG, Heredia V, Beltramo DM. Self-assembled micelles of monosialogangliosides as nanodelivery vehicles for taxanes. J Control Release. 2012;162(3):619-627.

26. Xu X, Li J, Han S, et al. A novel doxorubicin loaded folic acid conjugated PAMAM modified with borneol, a nature dual-functional product of reducing PAMAM toxicity and boosting BBB penetration. Eur $J$ Pharm Sci. 2016;88:178-190.

27. BremerEG, Hakomori S, Bowen-Pope DF, Raines E, Ross R. Gangliosidemediated modulation of cell growth, growth factor binding, and receptor phosphorylation. J Biol Chem. 1984;259(11):6818-6825.

28. Yu RK, Tsai YT, Ariga T, Yanagisawa M. Structures, biosynthesis, and functions of gangliosides - an overview. J Oleo Sci. 2011;60(10): 537-544.

29. Formisano S, Johnson ML, Lee G, Aloj SM, Edelhoch H. Critical micelle concentrations of gangliosides. Biochemistry. 1979;18(6): 1119-1124.

30. Corti M, Cantù L, Favero ED. Peculiarities in the aggregative behaviour of gangliosides, glucosidic surfactants of biological origin. IL Nuovo Cimento D. 1994;16(9):1391-1400.

31. Pan K, Chen H, Davidson PM, Zhong Q. Thymol nanoencapsulated by sodium caseinate: physical and antilisterial properties. J Agric Food Chem. 2014;62(7):1649-1657.

32. Fröhlich E. The role of surface charge in cellular uptake and cytotoxicity of medical nanoparticles. Int J Nanomedicine. 2012;7(11):5577-5591.

33. Beddoes CM, Case CP, Briscoe WH. Understanding nanoparticle cellular entry: a physicochemical perspective. Adv Colloid Interface Sci. 2015;218:48-68.

34. She W, Luo K, Zhang C, et al. The potential of self-assembled, pHresponsive nanoparticles of $\mathrm{mPEGylated} \mathrm{peptide} \mathrm{dendron-doxorubicin}$ conjugates for cancer therapy. Biomaterials. 2013;34(5):1613-1623.

35. Ma C, Pan P, Shan G, Bao Y, Fujita M, Maeda M. Core-shell structure, biodegradation, and drug release behavior of poly(lactic acid)/ poly(ethylene glycol) block copolymer micelles tuned by macromolecular stereostructure. Langmuir. 2015;31(4):1527-1536.

36. Neuhaus W, Gaiser F, Mahringer A, Franz J, Riethmüller C, Förster C. The pivotal role of astrocytes in an in vitro stroke model of the bloodbrain barrier. Front Cell Neurosci. 2014;8(6):352.

37. Roepe PD. Analysis of the steady-state and initial rate of doxorubicin efflux from a series of multidrug-resistant cells expressing different levels of P-glycoprotein. Biochemistry. 1993;31(50):12555-12564.

38. Goren D, Horowitz AT, Tzemach D, Tarshish M, Zalipsky S, Gabizon A. Nuclear delivery of doxorubicin via folate-targeted liposomes with bypass of multidrug-resistance efflux pump. Clin Cancer Res. 2000;6(5):1949-1957.

39. Ying X, Wen H, Lu WL, et al. Dual-targeting daunorubicin liposomes improve the therapeutic efficacy of brain glioma in animals. J Control Release. 2009;141(2):183-192.

40. Stojanov K, Georgieva JV, Brinkhuis RP, et al. In vivo biodistribution of prion- and GM1-targeted polymersomes following intravenous administration in mice. Mol Pharm. 2012;9(6):1620-1627.

41. Liang DS, Su HT, Liu YJ, Wang AT, Qi XR. Tumor-specific penetrating peptides-functionalized hyaluronic acid-D- $\alpha$-tocopheryl succinate based nanoparticles for multi-task delivery to invasive cancers. Biomaterials. 2015;71:11-23.

42. Adibhatla RM, Hatcher JF, Tureyen K. CDP-choline liposomes provide significant reduction in infarction over free CDP-choline in stroke. Brain Res. 2005;1058(1-2):193-197.

43. Grupp L, Wolburg H, Mack AF. Astroglial structures in the zebrafish brain. J Comp Neurol. 2010;518(21):4277-4287. 
44. Umans RA, Taylor MR. Zebrafish as a model to study drug transporters at the blood-brain barrier. Clin Pharmacol Ther. 2012;92(5):567-570.

45. Jeong JY, Kwon HB, Ahn JC, et al. Functional and developmental analysis of the blood-brain barrier in zebrafish. Brain Res Bull. 2008; 75(5):619-628.

46. Yang T, Martin P, Fogarty B, et al. Exosome delivered anticancer drugs across the blood-brain barrier for brain cancer therapy in Danio rerio. Pharm Res. 2015;32(6):2003-2014.

47. Guemez-Gamboa A, Nguyen LN, Yang $\mathrm{H}$, et al. Inactivating mutations in MFSD2A, required for omega-3 fatty acid transport in brain, cause a lethal microcephaly syndrome. Nat Genet. 2015;47(7):809-813.
48. Zhang B, Sun X, Mei H, et al. LDLR-mediated peptide-22-conjugated nanoparticles for dual-targeting therapy of brain glioma. Biomaterials. 2013;34(36):9171-9182.

49. Ruan S, Yuan M, Zhang L, et al. Tumor microenvironment sensitive doxorubicin delivery and release to glioma using angiopep-2 decorated gold nanoparticles. Biomaterials. 2015;37:425-435.

50. Desland FA, Afzal A, Warraich Z, Mocco J. Manual versus automated rodent behavioral assessment: comparing efficacy and ease of Bederson and Garcia neurological deficit scores to an open field video-tracking system. J Cent Nerv Syst Dis. 2014;6:7-14.
International Journal of Nanomedicine

\section{Publish your work in this journal}

The International Journal of Nanomedicine is an international, peerreviewed journal focusing on the application of nanotechnology in diagnostics, therapeutics, and drug delivery systems throughout the biomedical field. This journal is indexed on PubMed Central, MedLine, CAS, SciSearch ${ }^{\circledR}$, Current Contents ${ }^{\circledR} /$ Clinical Medicine,

\section{Dovepress}

Journal Citation Reports/Science Edition, EMBase, Scopus and the Elsevier Bibliographic databases. The manuscript management system is completely online and includes a very quick and fair peer-review system, which is all easy to use. Visit http://www.dovepress.com/ testimonials.php to read real quotes from published authors.

Submit your manuscript here: http://www.dovepress.com/international-journal-of-nanomedicine-journal 